\title{
Cooperación en el área de defensa en la UNASUR: un balance del Consejo de Defensa Sudamericano y sus límites
}

\author{
RUBÉN MIRANDA GONÇALVES \\ Doutorando em Direito Administrativo e Mestre em Direito das Adminis- \\ trações e Instituições Públicas pela Universidad de Santiago de Compostela \\ (Espanha). Professor do Mestrado em Seguridade, Paz e Conflitos Inter- \\ nacionais da Universidad de Santiago de Compostela (Espanha). Investiga- \\ dor contratado do Centro de Estudos em Seguridade da Universidad de \\ Santiago de Compostela (Espanha).
}

\section{MILTON CARLOS BRAGATTI}

\begin{abstract}
Mestre em Integração Regional Contemporânea pela UNILA e em Artes de Comunicação Internacional pelo New York Institute of Technology (Estados Unidos da América). Graduado em Integração Regional e Cooperação Sul-Sul pelo Consejo Latino-Americano de Ciencias Sociales (Argentina). Integrante do Núcleo de Estudos Estratégicos, Geopolítica e Integração Nacional (NEEGI) da Universidade Federal da Integração Latino-Americana pela UNILA.
\end{abstract}

Artigo recebido em 02/2/2018 e aprovado em 24/4/2018.

CONTENIDO: 1 Introducción - 2 El CDS y la defensa en la UNASUR: de iniciativa prometedora al riesgo de inflexión • 3 Los cuatro ejes de cooperación en el Consejo de Defensa de UNASUR: breve balance (2008-2012) - 4 Conclusión • 5 Referencias

RESUMEN: El área de defensa fue una de las que más avanzaron en el contexto de UNASUR, sin embargo, hay muchos desafíos y problemas para más cooperación en esa área. Si en los primeros años el Consejo de Defensa Sudamericano - CDS y la UNASUR obtuvieron éxitos, hay signos de estancamiento. El objetivo de este artículo es hacer un balance de la cooperación en Defensa en América del Sur dentro del CDS. Así, se busca comprender el proceso de creación del CDS y la complejidad y perspectivas que permean el área de la Defensa en América del Sur, sus logros y sus límites actuales.

PALABRAS CLAVE:UNASUR • Consejo de Defensa • Integración Regional • Cooperación Militar. 


\section{Cooperação na área de defesa da UNASUL: um balanço do Conselho de Defesa Sul-Americano e seus limites}

SUMÁRIO: 1 Introdução • 20 CDS e a defesa na UNASUL: de iniciativa promissora ao risco de retração - 3 Os quatro eixos de cooperação no Conselho de Defesa da UNASUL: breve balanço (2008-2012) • 4 Conclusão 5 Referências

RESUMO: A área de defesa foi umas das que mais avançou no contexto da UNASUL. Há, porém, muitos desafios e problemas que precisam ser enfrentados visando aumentar a cooperação nessa área. Se nos primeiros anos o Conselho de Defesa Sul-Americano - CDS e a UNASUL obtiveram êxitos, há agora sinais de estagnação. $O$ objetivo deste artigo é fazer um balanço da cooperação na área de defesa da América do Sul dentro do Conselho de Defesa Sul-Americano. Assim, busca-se compreender o processo de criação do CDS e a complexidade e as perspectivas que permeiam a área de defesa na América do Sul, bem como suas conquistas e seus limites atuais.

PALAVRAS-CHAVE: UNASUL • Conselho de Defesa • Integração Regional • Cooperação Militar.

\section{Cooperation in the defense area in UNASUR: a balance of the South American Council for Defense and its limits}

CONTENTS: 1 Introduction -2 The SCD and the defense in UNASUR: from a promising initiative to the risk of inflection 3 The four axes of cooperation in the UNASUR Defense Council: brief assessment (2008-2012) • 4 Conclusion $\bullet 5$ References.

ABSTRACT: The area of defense was one of the most advanced in the UNASUR context. However, there are many challenges and problems for further cooperation in this area. If in the early years the South American Council for Defense - SCD and the UNASUR achieved success, there are now signs of stagnation. The objective of this article is to take stock of the cooperation in Defense in South America within the SCD. Thus, it seeks the understanding of the process of creating the SCD and the complexity and perspectives that pervade the area of defense in South America, its achievements and its current limits.

KEYWORDS:UNASUR • Council for Defense • Regional Integration • Military Cooperation. 


\section{Introducción}

La inauguración de su moderno edificio en Quito, Ecuador, marcó en 2015, los ocho años de existencia de la Unión de Naciones Suramericanas - UNASUR. Además de la nueva sede, fue inaugurado también el mismo año de Escuela Suramericana de Defensa, la ESUDE, con la finalidad de formar cuadros militares y de funcionarios de Defensa con una visión compartida en el tema del sub continente. Desde su fundación, en estos 10 años, la UNASUR obtuvo sucesos en gerenciamiento y control de varias crisis políticas, como el desmantelamiento de tentativa de golpe en Ecuador, en 2010; la discusión sobre instalación y uso de bases militares colombianas por EEUU en 2009; y la mediación de la crisis entre oposición y gobierno en Venezuela en el 2014. Estas, entre otras situaciones, muestran que la actuación de la UNASUR podría representar un "subsistema político internacional diferenciado" en las Américas (PEÑA, 2009).

No obstante, aunque existen motivos para celebrarlo, hay elementos para preocuparse con una visible inflexión en el alcance de la entidad: el reciente apelo de Bolivia a la Corte de la Haya - y no a la UNASUR - para resolver la cuestión de la salida al mar en el litigio de la frontera con Chile; la negociación entre las Fuerzas Armadas Revolucionarias de Colombia - FARC y el gobierno de Colombia, en donde actores extra regionales asumieron protagonismo; y acuerdos bilaterales en defensa con países fuera de la región - notoriamente Rusia y China. Estos son algunos de los factores que enfatizan el momento delicado que el CDS-UNASUR atraviesa para que se firmasen y desarrollasen su potencial de entidad primordial en la resolución de esos asuntos.

El objetivo de este artículo consiste en hacer un breve balance de las acciones del CDS, los problemas y la complejidad y perspectivas que permean el área de Defensa en la UNASUR. Se van a analizar en este capítulo los fundamentos principales de la cooperación en defensa suramericana y el desarrollo de las iniciativas y proyectos fundamentales en el ámbito de CDS de la UNASUR, además del proceso de creación. Se presenta un breve sumario de los Planes de Acción del CDS, sus logros y limitaciones (Primer eje: políticas de defensa; Segundo eje: cooperación militar, acciones humanitarias y operaciones de paz; Tercer eje: industria y tecnología de defensa; Cuarto eje: formación y capacitación), más allá del trabajo realizado por el Centro de Estudios Estratégicos de Defensa - CEED y la creación de la Escuela Suramericana de Defensa. Finalmente, se apunta a los limites, dificultades y perspectivas para la cooperación en defensa en América de Sur. 


\section{El CDS y la defensa en la UNASUR: de iniciativa prometedora al riesgo de inflexión}

El área de Defensa fue una de las que más avanzaron en el contexto de la UNASUR y este es uno de los aspectos que la diferencian de otros procesos de regionalismo que desarrollaron otras áreas, como el comercio, y la defensa de la democracia. Además de los trabajos realizados por el Centro de Estudios Estratégicos de Defensa en Buenos Aires, la creación e inauguración de la Escuela Sudamericana de Defensa en Quito, son varias las acciones que refuerzan y estimulan la cooperación en defensa en el país, como la definición de Planes de Acción en el área de seguridad y defensa, la construcción de metodología común de medición de los gastos en defensa e intercambios en formación y capacitación militar (FUCCILLE, 2014).

Sin embargo, la UNASUR y en especial el CDS parecen atravesar un momento de inflexión, como la decisión de Bolivia de apelar a la Corte de La Haya - y no a la UNASUR - para la resolución de sus pleitos de salida al mar y tensión con el gobierno de Chile; la entrada de otros actores e instituciones en la mediación de las tensiones y conflictos entre la oposición y el gobierno venezolano y también las negociaciones de paz entre las FARC y el gobierno colombiano, en que la UNASUR no ha tenido un papel principal. Así mismo, en el ámbito de la industria de defensa, en los últimos años hay un fortalecimiento de acuerdos bilaterales entre países sudamericanos con socios extra-regionales, como China y Rusia, además de Irán, en el área de equipamientos militares. Todo ello en un contexto de reconfiguración geopolítica en el continente, con el restablecimiento de las relaciones entre EEUU y Cuba, teniendo en cuenta varios pleitos electorales en la región, que apuntan a un cambio de vientos en las iniciativas de integración y cooperación sudamericana.

Nos proponemos en este artículo a hacer un breve levantamiento de los avances institucionales de UNASUR y del CDS, y realizar un balance resumido de su desarrollo, en un contexto de reorientación política de varios países en el continente, a partir de las elecciones electorales recientes, más allá del contexto global de cambios políticos-institucionales. El CDS se basa en el respeto a la soberanía y no intervención y sus objetivos son: (1) consolidar la América del Sur como una zona de paz, libre de armas nucleares y de destrucción masiva; construir una identidad suramericana en materia de defensa; reforzar la cooperación regional en materia 
de defensa; y aumentar la defensa soberana de los recursos naturales de la región. El UNASUR se configura como un mecanismo relativamente flexible y eficaz para la gestión de crisis y, en algunos casos, la prevención de conflictos, en parte como una alternativa a la Organización de los Estados Americanos - OEA (SANAHUJA; VERDES-MONTENEGRO, 2014). Aunque existían contactos y acuerdos entre los países en esa área durante años, la arquitectura emergente que toma forma a partir de CDS representa la primera institucionalización de las cuestiones de seguridad en defensa en el ámbito estrictamente suramericano (SAINT-PIERRE, 2014).

\section{Los cuatro ejes de cooperación en el Consejo de Defensa de UNASUR: breve balance (2008-2012)}

El Consejo de Defensa de UNASUR es una iniciativa relativamente nueva, incipiente, caminando a pasos lentos y que aún no ha pasado por pruebas de fuego, especialmente con relación a desafiar frontalmente los intereses de potencias extra-regionales (FUCCILLE, 2014). Según Fuccille, "sólo fue posible erigir una estructura como el CDS por la opción de no confrontación abierta con Washington" (2014, p. 6, traducción nuestra). El autor argumenta que el CDS es un proceso hasta ahora en cierto modo consentido por Estados Unidos. Para desarrollar sus actividades en cooperación en defensa, el CDS ha establecido cuatro líneas principales de acción en el continente: (1) las operaciones de política de defensa; (2) la cooperación militar, la acción humanitaria y de paz; (3) industria y tecnología de defensa; y (4) formación y capacitación. En sus cinco primeros años, es posible realizar un balance relativamente positivo de las actividades de la entidad, a pesar de críticas tanto de académicos como de autoridades políticas y militares con lo que es percibido como vagarosidad en la implantación de sus resoluciones.

\subsection{Primer eje: políticas de defensa}

El primer eje, de políticas de defensa, es considerado el principal en las tres primeras ediciones del Plan de Acción del CDS. En este eje, la UNASUR y el CDS buscan desempeñar un papel en la afirmación de una visión estratégica común, de un enfoque regional para la seguridad, y una identidad sudamericana. Así mismo, aún existe un debate y cierta discordancia entre las distintas naciones sobre lo que se configura amenazas, prioridades y visiones estratégicas. En la visión de Sanahuja y Verdes-Montenegro (2014), se puede decir que hay un proceso de regionalización a partir de un proceso Marco común y una instancia 
de comunicación, socialización y aprendizaje llevada a cabo por el CDS. La implantación y el trabajo desarrollado por el Centro de Estudios Estratégicos de Defensa en el ámbito del Consejo de Defensa de América del Norte tiene como objetivo contribuir a la definición de enfoques estratégicos en la región y la articulación de una identidad de defensa sudamericana: los estudios publicados por la CEED hasta 2012, dos abordaron la concepción de seguridad y defensa en la región, observando la diversidad de visiones y estructuras institucionales existentes entre los doce países, según Sanahuja y Verdes-Montenegro (2014).

Un estudio elaborado por el CEED abordó también la protección de los recursos naturales y de la biodiversidad de la región, considerada estratégica, en la que una de las principales amenazas en la región, siendo rica en recursos, se relaciona, según Sanahuja y Verdes-Montenegro, a intentos de control extranjero sobre petróleo, agua y recursos minerales y agroalimentaria y, por lo tanto, que podrían estar sujetos a conflictos. Esta geopolítica de recursos naturales sugiere que la UNASUR busca construir un espacio geopolítico integrado que garantice el acceso y control de sus miembros de potenciales amenazas extra-regionales (2014). La publicación del registro sudamericano de gastos de defensa abre un nuevo camino de institucionalización en la medida en que los países miembros se reúnen y tienen noción clara de sus presupuestos y expectativa del informe anual de sus costos de defensa (FUCILLE, 2014; SANAHUJA; VERDES-MONTENEGRO, 2014; SAINTE-PIERRE; MONTOYA, 2014).

\subsection{Segundo eje: mantenimiento de la paz (cooperación militar, acciones humanitarias y operaciones de paz)}

El segundo eje de los Planes de Acción, sobre cooperación militar, acciones humanitarias y operaciones de paz, busca aliar las experiencias regionales de operaciones combinadas y conjuntas a través de entrenamientos comunes, y busca una forma de actuación conjunta y uso compartido de los mecanismos ya existentes. A través de los ejercicios interaliados UNASUR I, UNASUR II y UNASUR III, se busca a través del entrenamiento conjunto, disminuir las discrepancias de las diferentes doctrinas militares de las fuerzas armadas - FFAA sudamericanas (REZENDE, 2013).

La experiencia compartida entre varias naciones sudamericanas en sus experiencias en el envío de tropas a misiones de paz para la Organización de las Naciones Unidas - ONU fue aprovechada como factor de intercambio de informaciones y medidas de confianza entre los países de la región. La participación de América del Sur en las operaciones de paz no es reciente: desde la fundación de la ONU, Argentina, Brasil, Chile y Uruguay han enviado observadores militares para misiones 
de la organización en varias partes del mundo. Sin embargo, a partir de la década de 1990 (y especialmente después de los años 2000) esa participación alcanzó niveles inéditos, constando como región de mayor contribución en las misiones de paz de la ONU. Estos países sudamericanos identificaron que la participación en misiones de paz de la ONU es una parte integrante del compromiso con el mantenimiento de la paz y la seguridad internacional (SOUZA NETO, 2013). La decisión de participar en misiones de paz de la ONU tiene relación, entre otros factores, especialmente con la política exterior de cada país, según Aguilar (2011).

La participación en misiones de paz de la ONU ha sido un importante elemento de aproximación y cooperación en materia de seguridad y defensa para los países sudamericanos. Según Aguilar, hasta 2011 los Estados de América del Sur habían participado en 56 operaciones de la ONU y en aquel año, de las 16 operaciones en curso, 12 contaban con la presencia de países sudamericanos. Como ejemplo de centro de entrenamiento destacado en la región se puede citar el Centro Argentino de Entrenamiento Conjunto para Operaciones de Paz - CAECOPAZ, establecido en 1995; el Centro Conjunto para Operaciones de Paz de Chile - CECOPAC), iniciado en 2002; y el Centro Conjunto de Operaciones de Paz de Brasil - CCOPAB, creado en 2010. Los centros se especializaron para la formación necesaria para las operaciones de paz, además de desarrollar intercambios entre instructores y alumnos en el subcontinente (AGUILAR, 2011, LLENDERROZAS, 2007).

La Misión de Estabilización de las Naciones Unidas en Haití - MINUSTAH es considerada la de mayor nivel en cooperación entre los países sudamericanos hasta el momento. La MINUSTAH, fundada en 2004 y en funcionamiento hasta los días actuales, sirvió como escenario para niveles sin precedentes de coordinación y cooperación entre los Estados de América del Sur. Fue la primera misión en que países sudamericanos respondieron activamente con efectivos militares y civiles en una reunión crisis de país de la región. Además de países de América del Sur, se han sumado contingentes de países de América Central, creando una identidad latinoamericana en la operación (LLENDERROZAS, 2007; HIRST, 2007).

Souza Neto (2013) recuerda que, en 1996 los Estados miembros del Mercado Común del Sul - MERCOSUR decidieron crear un foro para la coordinación entre sus ministros de Relaciones Exteriores con el fin de promover posiciones comunes en instituciones internacionales, que se debió principalmente al Consejo de Seguridad de la ONU. La participación de estos países en la MINUSTAH también habría contribuido a acercar a los países contribuyentes 
con tropas - TCC (del acrónimo en inglés Troop Contributing Country): Argentina, Brasil, Chile, Perú y Uruguay fueron miembros activos del Grupo de Amigos de Haití de la ONU, siendo invitados a participar en reuniones del Consejo de Seguridad para discutir la situación en Haití y coordinar sus posiciones acerca de la renovación del mandato y cambios en sus prioridades de la misión. A raíz de la no participación de esos países en la historia de la colonización e intervención de Haití, a diferencia del caso de Francia e Inglaterra, estos Estados lograron una forma diferente de actuación con su contingente militar, buscando descartar la imagen de invasión y ocupación, intentando así una aproximación con la población local, por medio de actividades de carácter civil, como las Acciones Cívicas Sociales - ACISO (SOUZA NETO, 2010, traducción nuestra).

La cooperación sudamericana en Haití se benefició de factores tales como las anteriores iniciativas de cooperación; la jerarquía militar sin diferencias; la cultura y la proximidad de la lengua; y el intercambio de funcionarios para llevar a cabo cursos militares entre los diferentes centros de la región, lo que permite una estandarización en el conocimiento sobre las operaciones de paz (SOUZA NETO, 2010). Según Souza Neto "la participación en la MINUSTAH proporcionó el establecimiento de lazos entre Haití y los países de la región, disminuyendo el aislamiento en que Haití se encontraba y (re) integrando el país a la comunidad Latinoamericana" (2010, p. 50-51).

La Misión también generó ganancias en cooperación en seguridad y defensa en América del Sur. Entre las iniciativas regionales de influencia por la MINUSTAH, se puede destacar el mecanismo 2x9, así como la Asociación Latinoamericana de Centros de Entrenamiento para Operaciones de Paz - ALCOPAZ, la Fuerza de Paz Combinada Cruz del Sur, y el Consejo de Defensa Sudamericano - CDS, en el marco de UNASUR - como esfuerzos de la cooperación política y de seguridad y del deseo de plurilateralización de las acciones e implicación de mantenimiento de la paz (SOUZA NETO, 2010; KENKEL, 2013).

La ALCOPAZ es una asociación de centros de formación de mantenimiento de paz, una iniciativa presentada por Argentina e iniciada en 2008, con el objetivo de promover la eficiencia y la eficacia en el envolvimiento de América en las operaciones de paz. La asociación fue creada en agosto de 2008 y sus miembros actuales incluyen Argentina, Brasil, Chile, Colombia, Paraguay, Ecuador, Guatemala, Perú y Uruguay. Uno de los principales efectos de la asociación es intentar presentar una voz común en la Asociación 
Internacional de Centros de Formación para el Mantenimiento de la Paz - IAPTC, así como servir como foro para compartir lecciones aprendidas, intercambios entre los centros, iniciativas de investigación académica sobre las cuestiones relacionadas con las operaciones de paz y promover el intercambio de conocimiento entre los militares, la policía y los componentes civiles, con el objetivo de incentivar la estandarización de entrenamiento y procedimientos para seguir las directrices de la ONU (SOUZA NETO, 2013).

En el marco del CDS de la UNASUR, los objetivos que se relacionan con la cooperación de paz internacional incluyen: alcanzar una posición regional común en instituciones multilaterales; la promoción de la interoperabilidad de las fuerzas armadas; un aumento en la participación regional sobre la base de una doctrina común; y un compromiso de participar en misiones humanitarias (SOUZA NETO, 2010).

El primer seminario realizado del CDS para discutir cuestiones de misiones de paz se celebró en Montevideo en setiembre de 2010, donde los participantes decidieron crear un mecanismo para coordinar todas las áreas relacionadas con la participación en las operaciones, con el fin de evitar la duplicación de esfuerzos y de aumentar las capacidades de los Estados miembros para participar en tales misiones. Estos esfuerzos coordinados incluyeron ejercicios de mantenimiento de paz conjuntos (que ya existían antes de la MINUSTAH), seminarios e intercambios entre los centros de formación de operaciones de paz en la región.

La Fuerza de Paz Combinada Cruz del Sur es una iniciativa bilateral, formada por Argentina y Chile. El acuerdo de fuerza de paz combinada fue firmado en 2005. En él el entrenamiento y la preparación quedarían a cargo del Estado Mayor Conjunto, que cuidaría la implementación operacional. Una vez constituida la fuerza se pondrá a disposición de las Naciones Unidas, según el sistema United Nations Stand-By Arrangement System UNSAS, pudiendo ser desplazado con el pedido de la ONU y aprobación de los dos países. Al ver una iniciativa como ésta se puede constatar cómo una operación de paz como la MINUSTAH con sus niveles de cooperación e interacción, puede generar una medida de construcción de confianza mutua y de superación de rivalidades y desconfianzas entre países, teniendo en cuenta que Argentina y Chile tuvieron un historial de disputas territoriales en la década de 1980 (SOUZA NETO, 2010, p. 46). 


\subsection{Tercer eje: industria y tecnología de defensa}

La cooperación en el ámbito de la industria y las tecnologías de defensa es una cuestión importante para la UNASUR y el CDS al igual que a sus objetivos de autonomía regional, a partir del intento de reducir la dependencia de los proveedores tradicionales de Europa y Estados Unidos. Uno de los objetivos es desarrollar una industria regional de Defensa, que para asegurar su desarrollo es necesaria una escala internacional y tecnología propia, a través de la cooperación regional. Brasil es, por su dimensión y por la fuerza de su industria de armamentos, lógicamente el país con más capacidades e interés en desarrollar esta dimensión de la UNASUR. En el Plan de Acción 2010-2011, es el eje que registró más actividades (REZENDE, 2013; SANAHUJA; VERDES-MONTENEGRO, 2014).

EL CDS realizó una evaluación de industrias y tecnologías en la región, en la búsqueda de mantener un sistema integrado de información, un calendario de varias ferias, seminarios y eventos sobre el tema, además de promover seminarios de seguridad, de tecnología industrial básica y de defensa para incentivar la cooperación y el intercambio de experiencias (SANAHUJA; VERDES-MONTENEGRO, 2014). Además, otros dos proyectos importantes son el desarrollo y producción de una aeronave de entrenamiento básico - UNASUR I, liderado por Brasil y Argentina; y un sistema de Vehículos Aéreos No Tripulados - VANTs, liderado por Brasil, además de la participación de empresas de Chile y Argentina en el proyecto de la aeronave de transporte militar KC-390 (REZENDE, 2013; SANAHUJA; VERDESMONTENEGRO, 2014). Entre los países sudamericanos, sólo Argentina, Brasil y Chile desarrollaron una incipiente industria de defensa nacional. Como señala Tamires Souza, "Brasil puede ser considerado como el mayor productor sudamericano, debido principalmente a la base industrial creada durante la dictadura militar" (2015, p. 139, traducción nuestra). Sin embargo, Souza subraya que con el fin de la guerra fría, el escenario favorable para esos países sufrió cambios, como la elevación de la competencia entre industrias de defensa internacionales y, citando a Vilela, dice que "la reducción de la demanda de productos militares, sumada al decrecimiento las tarifas aduaneras resultantes de las presiones neoliberales y la falta de incentivo de los gobiernos, llevó a muchas industrias de defensa sudamericanas a la quiebra o a diversificar sus producciones" (VILELA, 2009, apud 
SOUZA, 2015, p. 139, traducción nuestra).

\subsection{Cuarto eje: formación y capacitación}

EL CDS ha desarrollado varias instancias en el área de formación y capacitación: además del Centro de Estudios Estratégicos de Defensa, en Buenos Aires, también fue creada e inaugurada la Escuela Suramericana de Defensa, con sede en Quito. El CEED es una instancia de producción de estudios estratégicos, un think tank, que tiene como misión la generación de conocimiento y difusión de un pensamiento estratégico sudamericano en términos de defensa y seguridad regional e internacional, siempre a iniciativa del CDS. El objetivo de la Escuela Suramericana de Defensa es promover y difundir consensos en las cuestiones estratégicas a través del diálogo.

El nuevo centro de estudios de la ESUDE fue inaugurado en 2015 y comienza a funcionar en la ciudad de Quito, capital de Ecuador y ciudad sede de UNASUR, encabezada por el brasileño Antonio Jorge Ramalho, asesor especial del Ministerio de Defensa y profesor de Relaciones Internacionales de la Universidad de Brasilia - UnB.

Otras acciones refuerzan y estimulan la cooperación en defensa en el continente, como la definición de Planes de Acción en el área de seguridad y defensa y la construcción de una metodología común de medición de los gastos militares en defensa, además de los intercambios en formación y capacitación militar (FUCILLE, 2014).

La inauguración y la implementación de la Escuela de Defensa Suramericana, a pesar de que aún no explicitar sus características y cómo será su funcionamiento, muestra que las iniciativas en ese sentido vienen caminando, consolidando la experiencia de los cursos CAD-SUR - los Cursos Avanzados de los cursos de la Defensa Suramericana - realizados por la Escola Superior de Guerra - ESG en años anteriores. Estas iniciativas de entrenamiento conjunto han crecido, aunque son pocas las iniciativas en ese sentido, y la mayor parte de ellas bancadas financieramente por Brasil (REZENDE, 2013).

Con formato descentralizado, la ESUDE es un centro de altos estudios responsable de la articulación de las diversas iniciativas de los países miembros del CDS para capacitación de civiles y militares en las áreas de Defensa y Seguridad Regional. En este sentido, el Centro de Estudios Estratégicos de Defensa fue creado en marzo de 2009 como órgano subordinado al CDS, que tiene como misión contribuir a la generación y propagación de un conocimiento estratégico sudamericano orientado a la defensa y a la seguridad Regional e internacional, contribuyendo y desarrollando una visión compartida por todos los 
países miembros de UNASUR (FRENKEL, 2016). De acuerdo con Frenkel, la ESUDE contempló principios de gradualismo y flexibilidad, en un diseño institucional descentralizado compuesto de una red de instituciones nacionales, cursos y disciplinas variadas, con una propuesta académica que puede ser individual para cada Estado miembro o en conjunto con otros países (FRENKEL, 2016, p. 45).

El Centro de Estudios Estratégicos de Defensa fue pensado como un órgano de producción académica para asesorar y proveer las informaciones necesarias al CDS. Su sede se encuentra en Buenos Aires, y corresponde a Argentina ceder el espacio físico - y se percibe que es ese país que viene teniendo más número de iniciativas para el desarrollo del CEED. Uno de los grandes desafíos a los que el CEED se propone, y aún no ha resuelto, es conceptual, sobre el papel y la función de las Fuerzas Armadas en nuestra región. La relación entre política interna y fuerzas armadas debe ser clara. En este sentido, las diferencias doctrinales de las FFAA sudamericanas obstaculizan la cooperación en defensa, al crear complicaciones, restringiendo así la cooperación (REZENDE, 2013).

\section{Conclusión}

El Consejo de Defensa de UNASUR, en pocos años de existencia, ha alcanzado un progreso significativo en el diálogo político. La institucionalización de un proceso de construcción regional de cooperación en Defensa contribuye a la confianza entre los Estados, favoreciendo la consolidación de la región como zona de paz. La construcción de medidas e instrumentos de confianza y de cooperación contribuyen a cambiar y/o al menos disminuir la percepción de rivalidad, tensión, desconfianzas e incluso la competencia entre los Estados. En este sentido, el intergubernamentalismo de la UNASUR ha sido conveniente para construir la confianza entre los Estados y el desarrollo institucional de la organización, de modo a favorecer la consolidación de una zona de paz, denotando un pragmatismo entre Estados renuentes a ceder soberanía en la toma de decisiones sobre políticas en organismos comunes. Sin embargo, ese intergubernamentalismo también puede ser responsable de los límites y dificultades enfrentados por el CDS en la formulación e implantación de sus proyectos y directrices (FUCCILLE, 2015, p. 115).

Los diversos cambios y turbulencias importantes en la política de varios países de América del Sur en el período a partir de 2015 - notablemente de énfasis conservador-neoliberal, como la elección de Maurício Macri, en Argentina; el proceso de impeachment de la presidenta Dilma Roussef, en Brasil; además de la evolución del 
proceso de paz en Colombia; y de ganancias electorales importantes de la oposición en Venezuela, entre otros, pueden traer también redefiniciones y reorientaciones potencialmente radicales en las estructuras y arreglos de regionalismo en la región, especialmente en la UNASUR, que tuvo su desarrollo bajo la de varios gobiernos populares de izquierda. Sin embargo, resaltamos que la evolución de la cooperación en Defensa en América del Sur demuestra que importantes acciones y arreglos fueron llevados a cabo por gobiernos de orientación neoliberal, como en la década de 1990 (auge del llamado Consenso de Washington), que sirvieron también como base inicial de varios desarrollos y mejoras, vistos en la conformación del Consejo de Defensa Suramericano. En este sentido, creemos que la cooperación en defensa entre las distintas naciones de la región debe seguir su curso incluso con incertidumbres, y, así como hay riesgos de regresión y disminución de la misma, no se puede descartar que esa misma cooperación también tenga posibilidad de aumentar, profundizar y desarrollarse bajo la orientación de otras fuerzas políticas, incluso con reformulaciones y redefiniciones.

\section{Referencias}

ABFIPONU - Associação Brasileira das Forças Internacionais de Paz. A participação brasileira nas forças de paz da ONU. Disponible en: <http://www.abfiponu.org.br/ historia02.html>. Acceso en: 24 jun. 2016.

AGUILAR, Sérgio Luiz Cruz. A participação Sul-Americana nas Operações de Paz da ONU: algumas considerações. Security and Defense Studies Review, v. 12, n. 1 e 2, p. 99-116, Fall-Winter 2011. Disponible en: <http://chds.dodlive.mil/files/2013/12/pub-SDSR-v12. pdf>. Acceso en: 8 mayo 2018.

.Cooperação e Coordenação: algumas considerações sobre a América Latina e a segurança internacional. In: Encontro Nacional da Associação Brasileira de Relações Internacionais, 4., 22 a 26 de julho de 2013, Belo Horizonte. Anais Eletrônicos... Belo Horizonte: ABRI, 2013. Disponible en: <http://www.encontronacional2013.abri.org.br/ download/download?ID_DOWNLOAD=498>. Acceso en: 24 jun. 2017.

CEED. Centro de Estudios Estratégicos de Defensa. Plan de Trabajo del Centro de Estudios Estratégicos de Defensa del Consejo de Defensa Suramericano 2012. Disponible en: <http://ceed.unasursg.org/Espanol/09-Downloads/Esp-PA/PTCEED-2012.pdf>. Acceso en: 8 mayo 2018.

.Centro de Estudios Estratégicos de Defensa.Plan de Trabajo del Centro de Estudios

Estratégicos de Defensa del Consejo de Defensa Suramericano 2013. Disponible en: 
<http://ceed.unasursg.org/Espanol/09-Downloads/Esp-PA/PT-CEED-2013.pdf>. Acceso en: 8 mayo 2018.

FRENKEL, Alejandro. Entre promesas y realidades: La UNASUR y la creación de la Escuela Suramericana de Defensa. Relaciones Internacionales, n. 31, Febrero 2016, Grupo de Estudios de Relaciones Internacionales - GERI. Madrid: UAM, 2016. Disponible en: <http://www.relacionesinternacionales.info/ojs/article/view/696. html>. Acceso en: 8 mayo 2018.

FUCCILLE, Alexandre. Conselho de Defesa Sul-Americano (CDS): Balanços e Perspectivas. In: Encontro da Associação Brasileira de Ciência Política, 9., 4 a 7 de agosto de 2014, Brasília, DF. Anais Eletrônicos... Brasília: ABCP, 2014. Disponible en: <https://cienciapolitica.org.br/index.php/system/files/ documentos/eventos/2017/04/conselho-defesa-sul-americano-cds-balanco-eperspectivas-714.pdf>. Acceso en: 8 mayo 2018.

FUCCILLE, Alexandre. O Brasil e a América do Sul: (re)pensando a segurança e a defesa na região. Revista Brasileira de Estudos da Defesa, ano 1, n. 1, jul./dez. 2015, p. 112-146.

HIRST, Monica. La intervención sudamericana en Haití. FRIDE Comentario, Madrid, 20 abr. 2007. Disponible en: <http://fride.org/publicacion/192/la-intervencionsudamericana-en-haiti>. Acceso en: 22 jun. 2016.

LLENDERROZAS, Elsa. Argentina, Brasil, Chile en la recontruccion de Haiti: interesses motivaciones de la participación conjunta. In: 2006 Meeting of the Latin American Studies Association. 15-18 March 2006, San Juan, Puerto Rico. Anais eletrônicos... San Juan, 2006. Disponible en: <http://www.resdal.org/producciones-miembros/artllenderrozas-lasamar06.pdf>. Acceso en: 21 jun. 2017.

LLENDERROZAS, Elsa. Los incentivos de Argentina, Brasil y Chile para participar en la misión MINUSTAH en Haiti. Su impacto en los mecanismos de cooperación en operaciones de paz.2007.93 f.Disertación final (Maestria en Relaciones y Negociaciones Internacionales) - Facultad Latinoamericana de Ciencias Sociales - Universidad de San Andrés en cooperación con la Universidad de Barcelona, Buenos Aires, Argentina, 2007. Disponible en: <http://flacso.org.ar/wp-content/uploads/2014/06/Disertacion. Elsa_LLenderrozas.03-04.pdf>. Acceso en: 21 jun. 2017.

PEÑA, F. La integracion del espacio sudamericano ¿La Unasur y el Mercosur pueden complementarse? Nueva Sociedad, n. 219, enero-febrero de 2009, p. 46-58. Disponible en: <http://nuso.org/media/articles/downloads/3582_1.pdf >. Acceso en: 8 mayo 2018.

REGUEIRO, Lourdes; BARZAGA, Mayra. UNASUR: proceso y propuesta. Quito: Fedaeps, 2012. 
REZENDE, Lucas Pereira. Sobe e desce! Explicando a cooperação em defesa na América do Sul: uma teoria realista-ofensiva. 2013. Tese (Doutorado em Ciência Política) - Universidade Federal do Rio Grande do Sul, Porto Alegre, 2013.

SAINT-PIERRE, Héctor Luis. La Defensa en la Política Exterior del Brasil: el Consejo Suramericano y la Estrategia Nacional de Defensa. Documento de Trabajo 50/2009. Madrid: Real Instituto Elcano, 2009. Disponible en: <http://www. realinstitutoelcano.org/wps/wcm/connect/1b12ab804fda364bb164ff8bf7fc 5c91/DT50-2009_Saint-Pierre_Defensa_Politica_exterior_Brasil.pdf?MOD=AJPER ES\&CACHEID=1b12ab804fda364bb164ff8bf7fc5c91>. Acceso en: 9 mayo 2018.

SAINTE-PIERRE, Héctor Luis; MONTOYA, Alberto (2014). As medidas de confiança no Conselho de Defesa Sul-americano (CDS): análise dos gastos em Defesa (2009-2012). Revista Brasileira de Política Internacional, Brasília, v. 57, n. 1, p. 22-39, 2014.

SANAHUJA, J. A.; VERDES-MONTENEGRO, F. J. Seguridad y Defensa en Suramérica: regionalismo, cooperación y autonomía en el marco de UNASUR. In: SERBIN, Andrés; MARTÍNEZ, Laneydi; RAMANZINI JÚNIOR, Haroldo. ¿Atlántico vs. Pacífico?: América Latina y el Caribe, los cambios regionales y los desafíos globales - Anuario de la Integración Regional de América Latina y ela Caribe No. 10, año 2014. Buenos Aires: Coordinadora Regional de Investigaciones Económicas y Sociales, 2014. p. 487-530. Disponible en: <http://eprints.ucm.es/39055/1/Anuario\%20CRIES\%202014\%20 Sanahuja\%20Verdes.pdf>. Acceso en: 9 mayo 2018.

SILVA, Peterson Ferreira. CT\&I e Defesa Nacional: novos rumos para o debate brasileiro? Revista Brasileira de Ciência, Tecnologia e Sociedade, v.2, n.1, p.239-251, jan/jun 2011. Disponible en: <http://www.revistabrasileiradects.ufscar.br/index. php/cts/article/viewFile/123/50>. Acceso em: 9 mayo 2018.

SOUZA NETO, Danilo Marcondes. Operações de Paz e cooperação regional: o Brasil e o Envolvimento sul-americano na MINUSTAH. Revista da Escola de Guerra Naval, Rio de Janeiro, n. 15, p. 25-58, 2010. Disponible em: <https://revista.egn.mar.mil.br/ index.php/revistadaegn/article/view/373/280>. Acceso em: 9 mayo 2018.

. Regional defense integration and peacekeeping cooperation in the Southern Cone. In: KENKEL, Kai Michael (Org.). South America and Peace Operations - Coming of Age, 1. ed., Londres: Routledge, 2013. p. 64-81.

SOUZA, Tamires Aparecida Ferreira. Cooperação em defesa e a região sul-americana: o papel do Conselho de Defesa Sul-Americano, da UNASUL. 2015. Dissertação (Mestrado em Estudos Estratégicos Internacionais) - Universidade Federal do Rio Grande do Sul, Porto Alegre, 2015. 
TORCHIARO, Luciana. MINUSTAH: una decisión estratégica con implicaciones regionales. Buenos Aires, 2007. Disponible en: <http://www.fes-seguridadregional. org/images/stories/docs/4110-001_g.pdf>. Acceso en: 24 jun. 2016.

TRIPODI, Paolo; VILLAR, Andrés. Haití: la encrucijada de una intervención latinoamericana. Revista Fuerzas Armadas y Sociedad, Año 19, n. 1 , p. 17-35, 2005.

UNASUR. Estatuto do Conselho de Defesa Sul-Americano. Santiago, 11 de dezembro de 2008. Disponible en: <http://www.defesa.gov.br/arquivos/relacoes_ internacionais/unasul/normativa_unasul_2017.pdf>. Acceso en: 9 mayo 2018.

.Plan de Acción 2010-2011. La Paz, 29 de julio de 2011. Disponible en: <http:// ceed.unasursg.org/Espanol/09-Downloads/Doc-Lineas/Metodologia_Comun.pdf>. Acceso en: 9 mayo 2018.

. Plan de Acción 2012. Disponible en: <http://ceed.unasursg.org/Espanol/09Downloads/Esp-PA/PA-CDS-2012.pdf>. Acceso en: 9 mayo 2018.

- Tratado Constitutivo de la Unión de Naciones Suramericanas. Quito, 11 de marzo 2011. Disponible en: <http://www.unasursg.org/images/descargas/ DOCUMENTOS\%20CONSTITUTIVOS\%20DE\%20UNASUR/Tratado-UNASUR-solo. pdf>. Acceso em: 22 mayo 2015.

VALES, Tiago Pedro. A participação sul-americana em operações de paz: uma estratégia regional de política externa? In: Colóquio Internacional de Doutorandos/ as do CES, 4., 6-7 de dezembro de 2013, Coimbra. Anais eletrônicos... Coimbra, 2013. Disponible en: <http://cabodostrabalhos.ces.uc.pt/n10/documentos/12.4.3_ Tiago_Pedro_Vales.pdf>. Acceso en: 24. jun. 2016.

VILELA, Fernando de Sousa. Integração das indústrias de defesa na América do Sul. Revista da Escola de Guerra Naval, Rio de Janeiro, n. 14, p.155-172, 2009. Disponible en: <https://revista.egn.mar.mil.br/index.php/revistadaegn/article/view/386/290>. Acceso en: 9 mayo. 2018. 